\title{
Gold-nanoparticle-based biosensors for detection of enzyme activity
}

\author{
Eliza Hutter and Dusica Maysinger
}

Department of Pharmacology and Therapeutics, Room 1314, Mclntyre Medical Sciences Building, McGill University, 3655 Promenade Sir William Osler, Montreal, QC, H3G 1Y6, Canada

\begin{abstract}
Optimal enzyme activity is essential for maintenance of physiological homeostasis. A variety of both nongenetic and genetic disruptions can excessively activate or silence intrinsic enzyme activities, with pathological outcomes. Many pharmacological agents are activators and inhibitors of enzymes. It is essential, therefore, in the development of drugs as enzyme activators and inhibitors, that enzyme activities be accurately measured under physiological and pathological conditions. Different biochemical assays have been developed for this purpose, some of which are based on nanostructured materials. This review focuses on gold nanoparticle (GNP)-based structures for the sensing and measurement of enzyme activities in biological specimens. Here we provide an overview and critical analysis of such assays, identify their advantages and limitations, and discuss interesting features of GNPs to be exploited for future applications in pharmacology.
\end{abstract}

\section{Gold nanoparticles in biosensing}

Gold nanoparticles (GNPs) have been used in the arts and medicine for centuries. They provided color for medieval cathedral windows and until the 18th century were believed to have life-prolonging and rejuvenating benefits if taken internally as aurum potabilis. Modern scientific evaluation of colloidal gold began with Michael Faraday's work in the 1850 s, which recognized that the color observed was due to the minute size of the gold. The technological advances of the 20th century made it possible to determine their exact morphological, physical, and chemical properties. In 1971, Faulk and Taylor were the first to use GNPs as immunocytochemical markers in transmission electron microscopy [1] . GNPs can be attached to many traditional biological probes such as antibodies, lectins, superantigens, glycans, nucleic acids, and receptors. Particles of different sizes are easily distinguishable in electron micrographs, allowing simultaneous multiple-labeling experiments. They can also be used for labeling thin cryosections for scanning electron microscopy, and samples for light microscopy. The unique properties of GNPs, such as their high absorption coefficient (see Glossary), scattering flux, luminescence, and conductivity, as well as their ability to enhance electromagnetic fields, quench (or enhance) fluorescence, and catalyze

Corresponding author: Maysinger, D. (dusica.maysinger@mcgill.ca).

Keywords: colorimetric assays; FRET; transferases; hydrolases;

matrix metalloproteinases.

0165-6147/\$ - see front matter

(c) 2013 Elsevier Ltd. All rights reserved. http://dx.doi.org/10.1016/j.tips.2013.07.002

\begin{abstract}
Glossary
Absorption coefficient: the absorption coefficient determines how far light of a particular wavelength can penetrate into a material before it is absorbed. Antibody: an antibody ( $\mathrm{Ab})$, also known as an immunoglobulin ( $\mathrm{lg})$, is a large Y-shaped protein produced by B cells that is used by the immune system to identify and neutralize foreign objects such as bacteria and viruses. Each tip of the $\mathrm{Y}$ of an antibody has a structure that is specific for one particular structure on its target, allowing these two structures to bind together with precision. As a consequence, an antibody can tag or neutralize its target directly (e.g., by blocking a part of a microbe that is essential for its survival and invasive character).

Avidin-biotin interaction: avidin is a protein that can bind up to four molecules of biotin simultaneously with a high degree of affinity and specificity. The strong affinity between avidin and biotin is widely used in biomedical research and industry.

Dipole moment: measure of net molecular polarity that can be defined as the product of the charge magnitude $(q)$ and the distance between the charges $(d)$. Förster resonance energy transfer (FRET): energy transfer between two chromophores, a donor and an acceptor, via a nonradiative mechanism without absorption or emission of photons.

Nanocage: particles of nanometer scale shaped in the form of a cage.

Quantum dots: nanometer-sized semiconductor particles composed of various compounds of elements such as cadmium, zinc, tellurium, selenium, and sulfur. Quantum dots react to electricity or light by emitting their own light across the visible wavelength range from 470 to $730 \mathrm{~nm}$. Quantum dots provide greater sensitivity and up to 1000 times brighter livecell imaging compared to conventional organic fluorophores. Quantum dots also have the advantage that they do not photobleach on exposure to light. In addition, quantum dots of different sizes can be excited by a singlewavelength light source, allowing simultaneous detection and multicolor imaging.

Quantum yield: the quantum yield of a fluorophore determines how much energy input is needed to produce a certain intensity of emitted light. It is defined as the ratio of the number of photons emitted to the number of photons absorbed.

Rayleigh scattering: elastic scattering of light or other electromagnetic radiation by particles much smaller than the wavelength of the light; named after the British physicist Lord Rayleigh.

Scattering flux: number of scattered particles per unit area per unit time.

Surface plasmon absorption peak: the wavelength at which maximum light absorption occurs due to the resonance conditions: matching between the natural frequency of surface electrons oscillating (surface plasmon) and the frequency of light photons.

Surface plasmon resonance (SPR): collective oscillation of electrons in solid or liquid stimulated by incident light. The resonance condition is established when the frequency of light photons matches the natural frequency of surface electrons oscillating against the restoring force of positive nuclei. SPR in nanometer-sized structures is called localized surface plasmon resonance.

Tat peptide: a domain of Tat protein that enters cells by crossing the cell membrane; it is therefore known as a cell-penetrating peptide. Tat protein is produced by a lentivirus (such as HIV) within infected cells. It greatly increases the rate of viral transcription and replication and is also secreted extracellularly, where it plays a role in increasing viral replication in newly infected cells and in enhancing the susceptibility of $T$ cells to infection.

Telomerase: enzyme that adds DNA sequence repeat units at the $3^{\prime}$ end of the DNA so that chromosomes are not shortened during cell division. Telomerase is particularly active in cancer cells.

Two-photon luminescence (TPL): emission of light after the simultaneous absorption of two photons of identical or different frequencies. The energy of the emitted photon is equal to the sum of the energies of the two photons.
\end{abstract}


reactions, provide numerous ways to exploit these particles for sensing purposes. Today, GNPs are used as catalysts and as imaging and therapeutic agents [2]. In addition, because of their remarkable versatility in biological and medical applications [3], GNPs have been proposed as signal transducers for biosensors [3].

Biosensors are analytical devices comprising two elements in spatial proximity: a biological recognition element, which is able to interact with the target, and a transducer, which converts the recognition event into a measurable signal. The myriad shapes and surfaces of GNPs available today, their relatively easy conjugation to molecules of interest, and their low toxicity are favorable features for biological applications. In biosensors, GNPs are the transducers, producing a measurable signal on biological recognition of the primary event occurring in close proximity, most often on their surfaces. GNP-based tests for various biomarkers are already commercially available (e.g., Nanosphere, Merck, and BBInternational), offering highly sensitive and specific detection of proteins and nucleic acids associated with infections, and with heart, kidney, and genetic diseases.

The life and death of complex organisms depend on the activity of enzymes. Enzymes are indispensable, for example, for regulation of signal transduction, for determining cell morphology function and differentiation, for adaptation to stressful stimuli, and for maintenance of cell homeostasis. The synthesis and activity of enzymes in living organisms strongly depend on the circumstances. For example, caspase 1, a key enzyme in inflammation and programmed cell death, exists mostly in its inactive precursor form in normal healthy cells. Cell exposure to whole pathogens or crystalline structures, such as asbestos, leads to the assembly of NLRP3 inflammasomes and subsequent activation of caspase 1 . Active caspase 1 processes the cytokines IL-1 $\beta$ and IL-18 and induces pyroptotic cell death. Measurements of enzyme activities and the posttranslational modifications they are subject to are important in the exploration of physiological and pathological processes (e.g., inflammation and cancer) and are crucial to enzyme research, enzyme engineering, and drug discovery. In traditional enzyme assays, catalytic activity is usually detected using labeled substrates or indirect sensor systems that produce a detectable spectroscopic signal on reaction. Although these approaches, and commercially available assays in general (e.g., GenScript), are adequate for many applications, they are limited in sensitivity and are restricted to in vitro use.

Advances in nanotechnology have now made it possible to measure minute changes in enzyme activity with high accuracy and precision. Nevertheless, few current nanoparticle-based assays are suitable for assessing enzyme-catalyzed transformations in vivo. The focus of this review is on the use of GNP-based assays for sensing enzyme activity in biological analytes (i.e., detection of enzyme-specific chemical reactions in biological environments). This detection depends on the transfer, removal, or addition of functional groups, the cleavage or joining of bonds, or the oxidation and reduction catalyzed by enzymes. Assays designed to quantify the amount of enzyme as a protein (rather than its catalytic activity) in a sample fall outside the scope of this review, but have been discussed elsewhere [2-4].

\section{GNP-based assays for enzyme activity detection}

Despite the many interesting physicochemical properties of GNPs, the majority of GNP-based enzyme assays exploit one of the following two GNP characteristics: (i) the surface plasmon absorption of GNPs; and (ii) their ability to quench fluorescence. Because of these properties, GNPs have been used in colorimetric-based and Förster resonance energy transfer (FRET)-based enzyme assays. In addition to colorimetric and FRET-based assays, other detection systems have been described for GNP-based enzyme activity determination, most notably electrochemical and light-scattering measurements.

\section{Colorimetric assays}

The surface plasmon absorption peak for GNPs is very sensitive to the environment, shifting towards longer wavelengths (red shift) and broadening significantly on GNP aggregation. In other words, the absorption peak for the dispersion changes towards longer wavelengths when the particles aggregate. This color change is easily detectable (Figure 1A). Conversely, the color shift can also occur in the reverse direction (Figure 1B); such a change may occur if the aggregated particles are linked to cleavable molecules. Cleavage of these molecules can be initiated by a change in the biological environment or an exogenous stimulus. These stimuli then cause the GNP aggregates to fragment into individual particles, leading to a blue shift in the absorption spectrum of the dispersion. The extent of aggregation/separation is proportional to the absorption peak shift, so the signal is quantifiable and provides a direct measure of the aggregation- or separation-inducing agent (e.g., an active enzyme) (Figure 1A,B). Biochemical assays based on color changes resulting from enhanced enzyme expression or changes in enzyme activity are popular in research and clinical laboratories because they are simple and do not depend on sophisticated instrumentation. However, these colorimetric assays can suffer from numerous problems owing to the presence of interfering molecules in biological samples that can absorb visible light in the same spectral region as the dispersed and/or aggregated particles. When this happens, it is difficult to distinguish the absorption peaks for GNPs and contributing biomolecules.

Colloidal gold is stabilized sterically or electrostatically by surface molecules. Aggregation or separation of GNPs due to enzyme activity can occur in several ways (Figure 1). An enzyme could compromise the stabilizing forces either by cleaving the surface molecules or by changing their charges, thus inducing aggregation of the particles. Linking the GNPs to antibodies recognizing the enzyme-modified surface molecule or using avidin-biotin interactions will also result in aggregation [5]. Conversely, interconnected (and therefore aggregated) GNPs can be separated when the link is enzymatically severed (Figure 1B).

Colorimetric assays are by far the most frequently used technique in GNP-based enzyme assays. The relatively simple measurement, inexpensive instrumentation, and robustness and potential for high-throughput assay make 


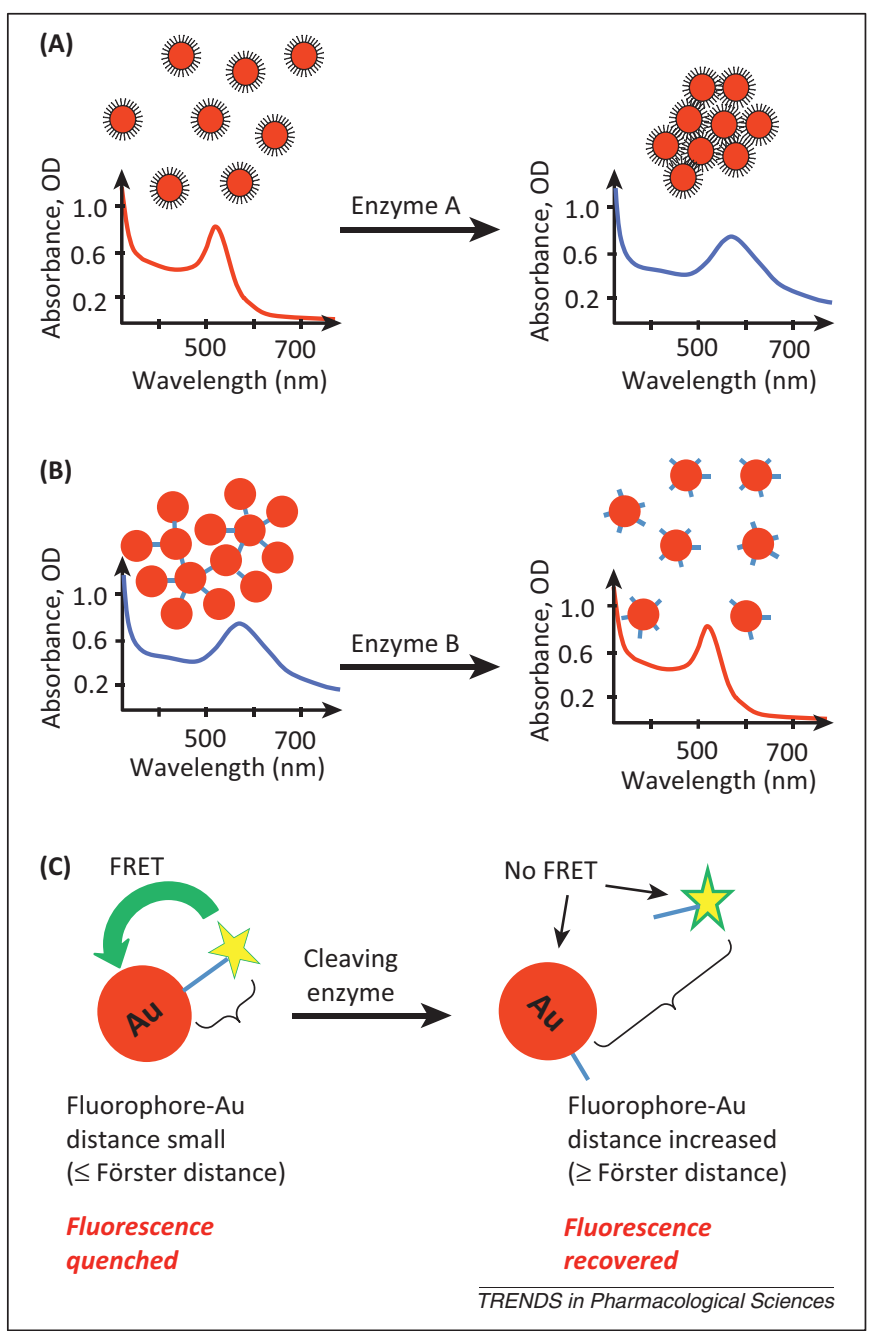

Figure 1. Principles of colorimetric detection and Förster resonance energy transfer (FRET)-based gold nanoparticle (GNP) enzyme assays. (A) Colloidal gold is stabilized sterically or electrostatically by surface molecules. Modification of the surface molecules by the enzyme compromises the stabilizing forces and thus induces aggregation of the particles (right) and consequently a blue shift of surface plasmon resonance peak. (B) GNPs, interconnected by molecules or forces that serve as a substrate for the enzyme (left), are separated by the enzyme action into individual particles (right), resulting in a red shift of the surface plasmon resonance peak. (C) The fluorescence of fluorophore $F$ (dye or quantum dot) is quenched in close proximity to GNPs and is recovered when the link is broken by the enzyme. The Förster distance is the distance at which the energy transfer efficiency is $50 \%$.

colorimetric GNP assays excellent candidates for in vitro measurements. There are already a number of colorimetric assays for transferases and hydrolases (Table 1). A typical example is the detection of histone-modifying enzymes [5]. In this assay, the surface of GNPs is functionalized with specific peptides derived from histone amino acid sequences that serve as substrates for histone methyltransferase or acetyltransferase. If these enzymes are present in the sample, then they will modify the peptides by adding methyl or acetyl groups. In the next step, specific sites within the short arms of the antibodies recognize methylated or acetylated peptide sequences and thus connect the GNPs and assemble them into network structures (i.e., quasi-aggregates). If the peptides are not methylated or acetylated by the enzyme, the antibodies do not bind to the unmodified peptides, aggregation does not occur, and there is no change in absorbance. Thus, aggregationdependent changes in absorbance are measured without the need for antibody labeling. This is the principle of label-free assays that utilize GNPs, which provides the specificity intrinsic to immunorecognition without chemical modification of the antibodies used. Most common biochemical assays use chemically modified antibodies with, for example, a fluorescent dye, peroxidase, biotin, or other labels, for determination of analytes in the absence of aggregation or cleavage processes or changes in electrical parameters.

Ratiometric quantification of enzyme activity is rarely used in colorimetric assays, even though it is a more reliable and sensitive approach than monitoring intensity changes at a single wavelength. Measurement of the ratio of the area under the surface plasmon peak for aggregated particles $(490-540 \mathrm{~nm})$ to the area for dispersed particles $(550-700 \mathrm{~nm})$ is an example, and has an extremely high detection limit of $90 \mathrm{zg} / \mathrm{ml}$ for thermolysin [6]. A similar approach could be used for a sensor consisting of GNPs assembled via protease-cleavable peptides and dispersed in the presence of the active enzyme. Such a sensor could easily be applied to the measurement of other proteases such as matrix metalloproteinases (MMPs). MMPs are hydrolases involved in many neurological disorders [7]. These proteases increase the permeability of the bloodbrain barrier as part of the neuroinflammatory response in hypoxia-ischemia, multiple sclerosis, and infection [7]. Recent studies have also implicated MMPs in the chronic neurodegeneration associated with vascular cognitive impairment, Alzheimer's disease, and Parkinson's disease. MMPs have key roles in tissue repair, in which they activate angiogenesis and neurogenesis. Therefore, both the identity of the active MMPs and their cellular origin could determine whether disease pathogenesis or regeneration occurs [7]. Owing to their clinical relevance, there is ongoing development of MMP-detecting assays [8]. Immunochemical methods are very common, but they cannot distinguish between active and inactive MMPs in zymogen form. Enzyme assays, using gelatine as a substrate, can measure MMP activity in both crude and purified samples of tumor tissue, and are suitable for inhibition studies; however, they cannot distinguish or separate isoenzymes with gelatinase activity. Array-based biosensors using modified gelatine overcame this issue [8]. Fluorimetric methods using fluorescently labeled substrates are particularly suitable for clinical use. They have low limits of detection (LODs), but they allow quantitative determination of MMP activity and they recognize the MMP sequences to be studied. Other methods [phage display, multiple-enzyme/multiple-reagent assay system (MEMRAS), activitybased profiling, radioisotopic, and electrochemical methods] are also being investigated [8]. In addition, highly sensitive, rapid GNP-based MMP assays have been developed [9]. The principle of these assays is detection of the proteolytic formation of oligonucleotide duplexes, which are used for signal amplification. In the presence of MMP-2, hydrolytic cleavage of DNA-peptide bonds on GNPs allows diffusion of DNA away from the GNP. This is followed by the formation of a DNA-RNA heteroduplex on another set of GNPs with fluorescently labeled RNA on their surfaces. Heteroduplex formation leads to RNA digestion by RNase H, which liberates it from the GNPs and recovers the fluorescence of the 
Table 1. GNP-based assays for detection of enzyme activity

\begin{tabular}{l} 
Enzyme activity detected \\
\hline Colorimetric assays \\
\hline Transferases \\
\hline
\end{tabular}

DNA adenine methylase and methylation sensitive

restriction endonuclease Dpn I

Endonuclease and methyltransferase

Telomerase

Histone methyltransferase and acetyltransferase

Kinase pKA and CaMK II

Kinases (pKA, pKC $\alpha$, CaMK II, MAPK-2, MAPAPK-2, c-Src)

$c-S r c$ tyrosine kinase

Protein kinase $\mathrm{A}$

\section{Hydrolases}

Alkaline phosphatase

Alkaline phosphatase

Endonuclease

Endonuclease and methyltransferase

Matrix metalloproteinase (MMP)

MMP-7

MMP-7

Heparinase

Thrombin, lethal factor

Thermolysin and nonbinding

R1-antichymotrypsin prostate-

specific antigen (nACT-PSA)

Trypsin or gelatinase

\section{Caspase 3}

$\beta$-Lactamase

S-Adenosylhomocysteine hydrolase

Lyases

Adenosine deaminase

LSD1 (lysine specific demethylase 1)

\section{Deamination of aden
citrate capped GNPs.}

Aggregation of GNPs in the presence of cleaved

aggregation of avidin-capped GNPs (methylated

Modified histones on the GNP surface are recognized
Detection limit $^{\mathrm{a}}$ peptide

Aggregation of GNPs in the presence of the product of enzyme activity

GNPs aggregate in the presence of cysteine, produced by the enzyme peptides are protected by antibodies)

Methylated DNA on the GNP is cleaved and the

GNPs interconnected with dsDNA separate on cleavage of the link by endonuclease; separation preventing aggregation Kinase reaction with biotinylated ATP results in GNP GNPs coagulate in the presence of nonGNPs aggregate in the presence of cationic

Aggregation of GNPs in the presence of dephosphorylated Tyr-Arg

dephosphorylated Tyr-Arg cleavage of the link

GNPs interconnected with dsDNA separate on methyltransferase inhibits the cleavage and subsequent separation the substrate is digested them

canometric detection of silver-enhanced GNPs on GNPs, reducing the signal

the enzyme degrades their surface coating

Uncleaved peptide induces GNP aggregation

GNPs linked to the enzyme substrate separate on cleavage of the link

GNP aggregation on digestion of their coating

Au nanocage and fluorescent dye linked together through enzyme cleavable peptides. Upon cleavage

fluorescence is recovered.
$0.3 \mathrm{U} / \mathrm{ml}$

I

HMT: $0.2 \mathrm{nM}$

HAT: $0.5 \mathrm{nM}$

$5.9 \mathrm{U} / \mathrm{ml}$

I,L

I

$\mathrm{I}, \mathrm{L}$

$0.01 \mathrm{U} / \mathrm{ml}$

I

$10 \mathrm{ng} / \mathrm{ml}$

I,B

$3 \mathrm{nM}$

$0.097 \mathrm{ng} / \mathrm{ml}$ (4.8 pM)

C

B

$5 \mathrm{nM}$ and $25 \mathrm{nM}$

$90 \mathrm{zg} / \mathrm{ml}$ and $10 \mathrm{ag} / \mathrm{ml}$

Trypsin: 0.125-125 U

Gelatinase:

$50-600 \mathrm{ng} / \mathrm{ml}$

10 and $5 \mathrm{ng} / \mathrm{ml}$

L

$\mathrm{I}, \mathrm{X}$

$0.1 \mathrm{U} / \mathrm{ml}(6 \mathrm{nM})$

I

$0.0008227 \mathrm{U} / \mathrm{ml}$

$13 \mathrm{pM}$
FRET-based assays

Hydrolases

MMP-2 
Table 1 (Continued)

\section{Enzyme activity detected}

MMP2

MMP-2

MMP-2

MMP-7

Protease uPA (urokinase-type plasminogen activator), Her-2 kinase (human epidermal growth factor receptor 2 )

Protease (trypsin)

Thrombin

Caspase 3 , caspase 8 , caspase 9

Caspase 3

Caspase 3

MMP-7, caspase 3, and thrombin

Thrombin, thermolysin, cathepsin L, caspase 3

MMP-2

Other assays

Oxidoreductases

Superoxide dismutase

Transferases

DAM (DNA methyltransferase)

Protein kinase $\mathrm{A}$

Protein kinase A

Protein kinase A

Protein kinase $\mathrm{C}$, protein tyrosine kinase

Protein kinase A

Tyrosine Abl kinase and PKA

Tyrosine Abl kinase
Assay principle

MMP-2 cleavage frees the highly fluorescent

photosensitizer from the gold nanorods

GNPs quench the fluorescence of a fluorophore by

being linked to it through the substrate for MMP2.

Upon cleavage quenching is eliminated and the

fluorescence restores

Recovery of bioluminescence after cleaving the peptide connecting luciferase and GNPs

Dye-tethered peptides are coordinated to GNPs, resulting in quenched fluorescence; dequenching occurs on cleavage

UPA: dequenching of QD fluorescence when substrate is cleaved

Her-2: FRET from QD to fluorophore when the fluorescently labeled antibody recognizes the phoshorylated substrate on the QD

Dequenching of the fluorescence of GNP-bound dye by bond cleavage

GNPs quench the fluorescence of a fluorophore via linkage through the protease substrate; on cleavage, quenching is eliminated and the fluorescence is restored

Dequenching of fluorescent proteins when their link to GNP is cleaved

Dequenching of fluorescent dye molecules when their link to GNPs is cleaved

Dequenching of fluorescent dye molecules when their link to GNP-containing nanogel is cleaved

Fluorescence recovery of QDs when GNPs are cleaved

Dequenching of fluorescent dye molecules when their link to GNPs is cleaved

Multiple step assay, ending with fluorescence recovery of labeled RNAs, when freed from GNPs

\section{Detection limit $^{\text {a }}$}

Sample $^{\text {b }}$ Refs

C [56]

$\mathrm{I}, \mathrm{A}$

$50 \mathrm{ng} / \mathrm{ml}$ to $1 \mu \mathrm{g} / \mathrm{ml}$

$10 \mathrm{ng} / \mathrm{ml}$

uPA

$50 \mathrm{ng} / \mathrm{ml}$

Her-2 kinase $7.5 \mathrm{nM}$

A

$0.1 \mathrm{nM}$

I,C

I,C

I,C,T

MMP-7: $10 \mathrm{ng} / \mathrm{ml}$ to $5 \mu \mathrm{g} / \mathrm{ml}$

Caspase 3: $20 \mathrm{ng} / \mathrm{m}$

Thrombin: $1 \mathrm{U} / \mathrm{ml}$

$10 \mathrm{pM}$

Electrocatalytic activity of a cytochrome $c$-nanogold

$0.25 \mathrm{U} / \mathrm{ml}(50 \mathrm{ng} / \mathrm{ml})$ complex changes in the presence of superoxide radical

Electrochemical assay; GNPs are hybridized to the

$0.2 \mathrm{U} / \mathrm{ml}$ electrode through DNA, which, if methylated, is cleaved by an endonuclease causing GNP detachment and generating an electrochemical signal

Electrochemical detection of DNA-modified GNPs bound to immobilized substrates at phosphorylation sites through $\mathrm{Zr}^{4+}$ ions

GNP-based electroluminescence probes are linked to magnetic beads through $\mathrm{Zr}^{4+}$ ions (if phosphate groups are present) and enhance the ECL signal Electrogenerated chemiluminescence; GNPs attach to phosphorylated kemptide and enhance the ECL signal Electrochemical detection of GNPs, bound to immobilized substrate peptides phosphorylated with a thiophosphate group

Labeling of specific phosphorylation events on a microarray with GNPs using avidin-biotin chemistry followed by silver enhancement and RLS detection Secondary-ion mass spectrometric imaging; SI ratiometry between unphosphorylated and phosphorylated signals for substrates bound to GNPs

Phosphorylated peptides on GNP monolayers are detected by an antiphosphotyrosine antibody and Cy3-labeled secondary antibody as a probe

\begin{tabular}{|l|l|l|}
\hline $0.15 \mathrm{U} / \mathrm{ml}$ & $\mathrm{I}$ & {$[66]$} \\
\hline $0.005 \mathrm{U} / \mathrm{ml}$ & $\mathrm{I}$ & {$[33]$} \\
\hline $0.07-32 \mathrm{U} / \mathrm{ml}$ & 1 & {$[19]$} \\
\hline $10 \mathrm{ng} / \mathrm{ml}$ & 1 & {$[67]$} \\
\hline & 1 & {$[68]$} \\
\hline
\end{tabular}

$50 \mathrm{pg} / \mathrm{ml}(1 \mathrm{pM})$ to $10 \mathrm{ng} / \mathrm{ml}$ । 
Table 1 (Continued)

\begin{tabular}{|c|c|c|c|c|}
\hline Enzyme activity detected & Assay principle & Detection limit $^{\mathrm{a}}$ & Sample ${ }^{b}$ & Refs \\
\hline Telomerase & $\begin{array}{l}\text { The product of telomerase activity is captured on a } \\
\text { gold electrode and reacted with signal DNA on GNPs; } \\
\text { Ru ions bind to the DNA and generate a } \\
\text { chronocoulometric signal }\end{array}$ & & C & [18] \\
\hline T4 polynucleotide kinase & $\begin{array}{l}\text { Substrate for kinase is immobilized on the electrode; if } \\
\text { phosphorylated by the enzyme, } \mathrm{TiO}_{2} \text { nanotubes, GNP- } \\
\text { modified } 5^{\prime} \text {-phosphate DNA and labeled signal DNA } \\
\text { assembles on top, generating an electrochemical } \\
\text { signal }\end{array}$ & $0.003 \mathrm{U} / \mathrm{ml}$ & 1 & [70] \\
\hline Alkaline phosphatase (ALP) & SERS detection of the product of ALP & $4 \mathrm{aM}$ & & [71] \\
\hline ALP & $\begin{array}{l}\text { Resonance scattering increases, as more gold is } \\
\text { formed with the product of ALP }\end{array}$ & $0.00003 \mathrm{U} / \mathrm{ml}$ & B & [72] \\
\hline $\begin{array}{l}\text { Subtilisin A (non-specific } \\
\text { protease), asparaginase, } \\
\text { glutamase, glutamate } \\
\text { carboxypeptidase II, caspase } 1\end{array}$ & $\begin{array}{l}\text { Uncleaved substrate inhibits catalytic activity of the } \\
\text { GNP-attached molecule and production of the colored } \\
\text { reporter molecule }\end{array}$ & Nanomolar & & [73] \\
\hline Trypsin & $\begin{array}{l}\text { Digestion of peptides covering the electrode results in } \\
\text { increased access to it by electrochemical probes and } \\
\text { generates an increase in electrochemical signal }\end{array}$ & $0.026 \mathrm{U} / \mathrm{ml}$ & & [75] \\
\hline
\end{tabular}

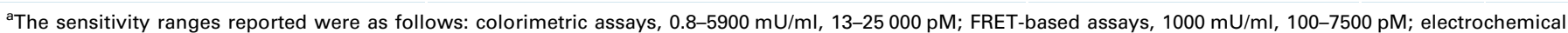
assays, $5-250 \mathrm{mU} / \mathrm{ml}, 0.8 \mathrm{pM}$; and resonance light scattering, $0.03 \mathrm{mU} / \mathrm{ml}$.

${ }^{\text {b}}$ Samples tested with: I, enzyme inhibitors; L, cell lysates; C, cell cultures; B, blood; X, bacteria; A, live animals; and T, tumor model.

RNA label. Because of the high quenching efficacy of GNPs for the fluorophore in RNA and multiple RNA digestions, the fluorescence signal recovery is amplified. This method can be used to assess MMP-2 activity at concentrations as low as $10 \mathrm{pM}$ within $4 \mathrm{~h}$. Compared to protease nanosensors using quantum dots (QDs), GNPs, and magnetic NPs with the same peptide sequence, the assay time for the oligonucleotide duplex method is six-fold shorter and the LOD is 100fold lower [9].

Highly sensitive methods based on GNPs for measurement of specific MMP activities could serve as diagnostic tools for the above-mentioned neurological disorders and the development of synthetic MMP inhibitors for therapeutic interventions. The advantage of GNP-based MMP measurements over most common colorimetric assays is their extremely high sensitivity.

\section{FRET-based assays}

FRET is energy transfer between two chromophores, a donor and an acceptor, via a nonradiative mechanism without absorption or emission of photons. One of the main requirements for FRET occurrence is close proximity of the donor and acceptor so that energy is transferred rather than dissipated in the environment. The distance between the two chromophores must be less than $10 \mathrm{~nm}$ because of the huge decrease in energy transfer with distance $\left(1 / R^{6}\right)$ between the donor and acceptor. Another important requirement for FRET is overlap between the donor emission and acceptor excitation spectra. Finally, the relative orientation of the dipole moments of the chromophores also influences the FRET efficiency: the highest FRET occurs for parallel dipole orientation. FRET-based assays have often been used to study receptor-ligand interactions [10]. For example, FRET between two different labeled receptor-bound fluorescent ligands has been used to detect oligomeric G-protein-coupled protein (GPCR) complexes in transfected cells and in native tissue [11]. Specific FRET signals were observed for four different receptors expressed in cell lines, consistent with their dimeric or oligomeric nature in these transfected cells. In tissues, the presence of oxytocin receptor dimers and/or oligomers in mammary gland has been demonstrated [11].

In FRET-based enzyme assays, GNPs act as acceptors, quenching the emission of donor chromophores (Figure 1C). Because of their large absorption cross-section, GNPs have a superior quenching efficiency for a broad wavelength range compared to other organic quenchers [12]. Because they have no defined dipole moment, energy transfer occurs for any orientation of the donor relative to the GNP surface, which increases the probability of energy transfer and accounts for the enhanced efficiency. GNPs as acceptors for energy transfer are superior to organic quenchers in two significant ways: (i) the distance dependence changes from $1 / R^{6}$ to $1 / R^{4}$, which extends the usable distances for measurement; and (ii) the same nanoparticle is able to quench dyes of different emission frequencies, from the visible range to the near-IR. Consequently, GNPs can be used for studies in which distances greater than $10 \mathrm{~nm}$ are expected or in which multiple dyes need to be quenched. It is also likely that a single nanoparticle has the ability to 
quench several dyes of different energies simultaneously, thereby allowing analysis of several target molecules in a single experiment [13].

Predictably, enzyme assays based on fluorescence quenching by GNPs are designed exclusively for the enzymes that cleave their substrates. Severing the link between the GNP and the fluorophore, thereby restoring the fluorescence of the fluorophore, is the basic detection principle in these assays (Figure 1C, Table 1). However, it is important to take into consideration that the fluorescence intensity of the free dye strongly depend on the GNP concentration in solution and on the spectral overlap between fluorophore emission and the GNP surface plasmon absorption peak [14]. In one study, for example, fluorescent dyes were attached to the surface of gold nanocages via protease cleavable peptides [14]. In the presence of the protease, the peptides were cleaved, the dye molecules were released from the surface of the nanocage, and the fluorescence was recovered. The surface plasmon absorption peak of gold nanocages can be tuned by changing their dimensions, and better detection sensitivity was observed when the localized surface plasmon peak of the nanocages was far from the fluorophore emission peak.

One of the most important advantages of fluorescent assays is that they allow in vivo imaging. In an elegant example, a near-infrared-fluorescence-quenched GNP imaging probe, capable of detecting MMP-2 activity, was injected into murine SCC7 (squamous cell carcinoma) tumors [15]. Tumors, with high protease activity, were clearly visualized by the near-infrared fluorescence signal from the probe. Pretreatment with a protease inhibitor reduced the tumor contrast significantly [15].

\section{Other assays}

Alternative assays to those based on absorbance or FRET are summarized in Table 1. Enzyme activity is detected via changes in the scattering intensity or electrochemical signal or via other methods. Light scattering by GNPs is most commonly visualized by dark-field microscopy. This reveals the individual ultrabright, nonbleaching GNPs, whose scattering intensity changes enormously with their size. (The scattering cross-section of a GNP of radius $R$ varies with $R^{6}$.) [16]. Alivisatos' research group exploited this outstanding feature in a study in which cleavage was monitored by caspase 3 , an enzyme implicated in the onset of apoptosis [17]. Their so-called crown nanoparticle plasmon rulers consisted of five satellite GNPs connected to a core GNP via a caspase-3-cleavable peptide linker (Figure 2). Each cleavage resulted in elimination of one of the satellite particles and a measurable decrease in scattering intensity, detected by a dark-field microscope equipped with a spectrometer. The caspase 3 activity in live cells was continuously monitored for over $2 \mathrm{~h}$, sufficient time to observe early-stage caspase 3 activation [17].

A variety of electrochemical assays can be used for GNPbased detection of enzyme activity. Cyclic voltammetry, differential pulse voltammetry, square-wave voltammetry, chronocoulometry, and impedance spectroscopy are used to detect current and charge responses to changes induced by enzyme action. GNPs are usually included in sensor structures as signal amplifiers. For example, the activity of human telomerase in cell lysates was determined using chronocoulometry (Figure 3) [18]. Telomerase is an enzyme that adds DNA sequence repeat units at the $3^{\prime}$ end of the DNA. In that study, cell lysates were mixed with the primer and nucleotides necessary for the enzyme to form longer single-strand DNA. This extension product was hybridized with a capture DNA immobilized on the electrode and then reacted with the signal DNA on GNPs. An electrochemical signal was generated by chronocoulometric measurements of $\left[\mathrm{Ru}\left(\mathrm{NH}_{3}\right)_{6}\right]^{3+}$, which quantitatively binds to the DNA on GNPs via electrostatic interaction. This method could detect the telomerase activity from as few as ten cultured cancer cells (Figure 3) [18].

In another study, tyrosine kinase-catalyzed phosphorylation of peptides on an electrode was detected using

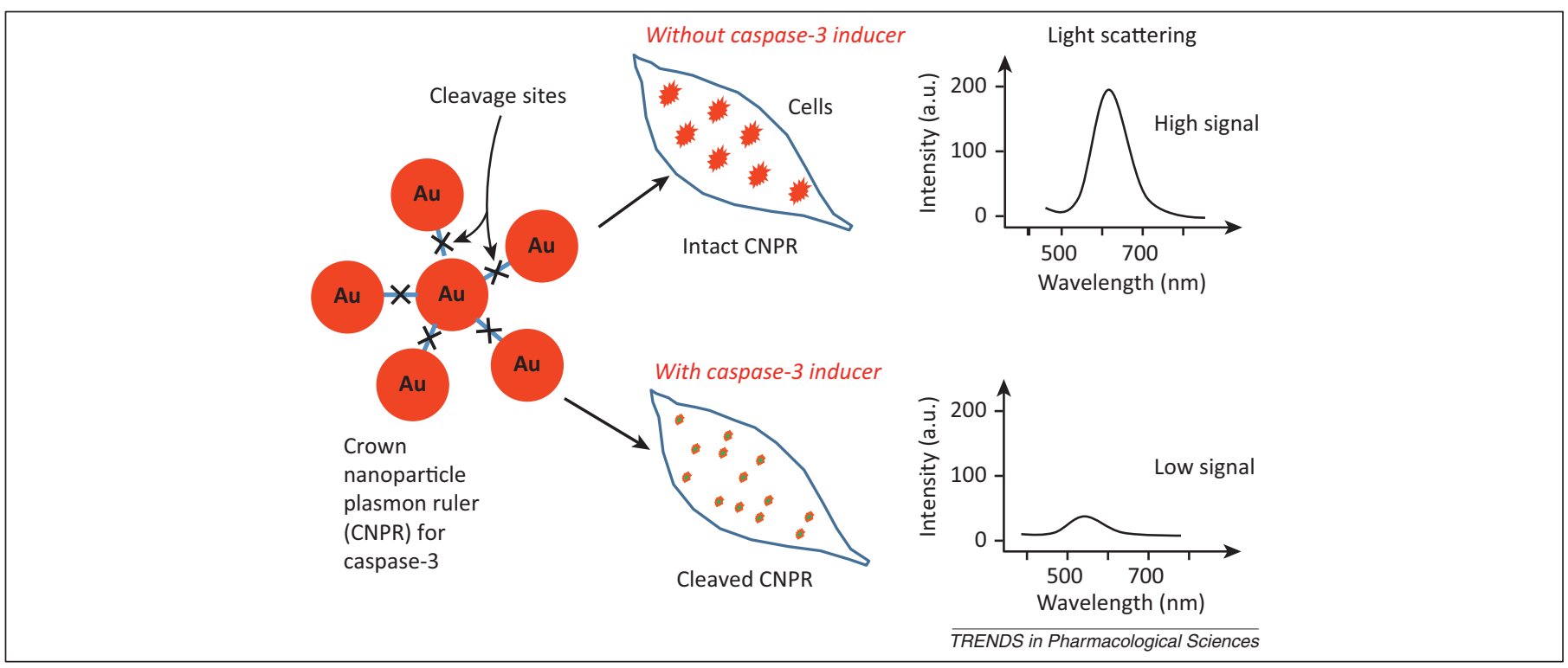

Figure 2. Detection of caspase 3 activity in live cells by light-scattering intensity measurements. Crown nanoparticle plasmon rulers are composed of one core and five satellite gold nanoparticles (GNPs), linked by a caspase-3-cleavable peptide. In the presence of active caspase 3, the rulers break up into individual nanoparticles and the light-scattering intensity decreases (compare the graphs at the top and bottom). Not drawn to scale. 


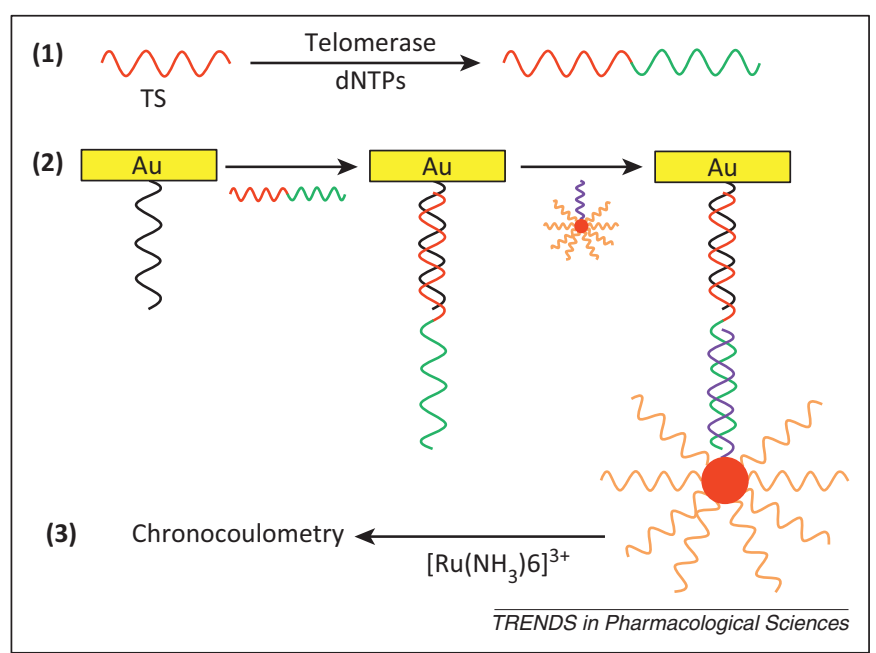

Figure 3. Principle of electrochemical detection of telomerase activity. Telomerase was extracted from HeLa cells. 1. The extract was mixed with telomerase substrate (TS) primer and nucleotide mixture (dNTPs) to perform extension reaction. 2. The extension product was hybridized with the capture DNA (black) immobilized on the Au electrode and then reacted with the signal DNA (blue) on $\mathrm{Au}$ nanoparticles to form a sandwich hybridization mode. 3. Electrochemical signals were generated by chronocoulometric measurements of binding of $\left[\mathrm{Ru}\left(\mathrm{NH}_{3}\right) 6\right]^{3+}$ to the DNA on Au nanoparticles via electrostatic interaction.

adenosine $5^{\prime}$ - $[\gamma$-thio] triphosphate (ATP-S) as a cosubstrate [19]. On thiophosphorylation of the peptide, GNPs accumulated on the surface (binding to the thiol group) and were detected by square wave-voltammetry by monitoring $\mathrm{Cl}^{-}$ oxidation at the GNP surface [19]. A similar concept with a different detection system was used to profile kinase activity with an electrogenerated chemiluminescence (ECL) biosensor [20]. In ECL, also known as electrochemiluminescence, electrochemically generated intermediates undergo a highly exergonic reaction to produce an electronically excited state that then emits light. ECL has several attractive features, including the absence of a background signal, precise control of reaction kinetics offered by control of the applied potential, compatibility with solution-phase and thin-film formats, and opportunities to enhance intensity with nanomaterials such as metallic nanoparticles and nanotubes. Together, these features make ECL a highly sensitive and selective analytical method [21]. $\mathrm{Xu}$ et al. demonstrated the use of ECL for assaying protein kinase A [20]. GNPs were assembled on an electrode via introduction of thiol groups during kinase-catalyzed phosphorylation in the presence of ATP-S. The assembled GNPs then mediated the ECL reaction of luminol, substantially amplifying the ECL signal by providing a large electrode-solution interface area and facilitating electron transfer between luminol and the electrode. The biosensor exhibited high sensitivity, a low detection limit of $0.07 \mathrm{U} / \mathrm{ml}$, a wide linear range (0.07-32 U/ml), and excellent stability [20]. In comparison, commercially available immunoassays have a detection limit of $5 \mathrm{U} / \mathrm{ml}$ (http://www.arborassays.com/ documentation/inserts/K027-H.pdf).

\section{Challenges in GNP-based nanosensor development}

The requirements for a useful enzyme assay are specificity, sensitivity, robustness, high-throughput potential, cost efficiency, and convenience. At present, GNP-based enzyme assays are in the research phase and their future potential regarding these requirements can only be estimated from published information. The type of application (e.g., sophisticated scientific investigations vs mass screening of analytes) also determines the requirements that are most important and hence which approach is the most suitable. For example, colorimetric assays have high potential for high-throughput applications and they are robust, simple, inexpensive, and require minimal instrumentation. The detection limits of GNP-based assays are surprisingly low, suggesting remarkable sensitivity (Table 1). Overall, colorimetric assays are very promising for assessing the effectiveness of enzyme inhibitors as potential drugs $[22,23]$. Such enzyme inhibitors could be used for therapeutic interventions in pathologies characterized by excessively active enzymes. In addition, colorimetric assays are useful for routine determinations of enzyme activity in biological specimens to distinguish between physiological and pathological states. It is unlikely however, that colorimetry can be easily adapted for complex biological environments (tissues or living organisms) because of the substantial interference from cellular macromolecules.

For measurement of enzyme activity via GNPs in cells, tissues, and whole animals, FRET-based assays show more promise than colorimetric assays (Table 1) [24-26]. Unexpectedly, the sensitivity reported for FRET-based assays is often lower than that reported for other assays, but this shortcoming could be remedied by appropriate modification of the selected materials or experimental design. The signal-to-noise ratio and therefore the sensitivity could be significantly improved by choosing dyes or QDs with higher fluorescence intensity. The sensitivity of FRET assay can also be improved by reducing background fluorescence with the most efficient quencher and operating in a wavelength range away from cellular autofluorescence.

In practice, biosensors are exposed to biomolecules, cells, tissues, and ultimately to whole organisms, and inevitably interact with these complex environments. The possibility of nonspecific binding and ligand exchange should be taken into account when designing a biosensor [11]. In the case of GNPs, which are usually functionalized with thiolated ligands using the strong thiol-metal bond, ligand exchange may occur in the presence of high concentrations of other thiolated molecules (e.g., intracellular glutathione) [27]. In addition, many of the ligands are charged owing to their functional groups and are therefore likely to interact nonspecifically with biomolecules. One way to prevent ligand exchange and nonspecific binding is to use a mix matrix, incorporating a defined amount of PEGylated alkanethiol in the matrix [28]. PEGylated alkanethiol ligands impart high stability and low nonspecific binding to metal nanoparticles. The ethylene glycol group is hydrophilic, but is not charged at physiological $\mathrm{pH}$; the stability imparted to the nanoparticle is probably due to the high flexibility of the ethylene glycol groups, preventing aggregation. It has been shown for a caspase 3 protease sensor that the mix matrix method provides remarkable resistance against ligand exchange while fully preserving its function [29].

Although GNP-based biosensors for in vitro determination of enzyme activities are certainly useful, there are still 
limitations. For example, the majority of GNP-based assays are easily used in vitro but the constructs cannot enter cells (e.g., because of the size limitations). However, GNPs with Tat peptides can easily penetrate living cells [17] but could induce some toxicity in certain cell types [30]. Clearly, monitoring of enzyme processes in vivo in an intact organism is still a challenge owing to the lack of knowledge on GNP biodistribution and elimination [31]. Even if GNPs cannot be used for non-invasive detection of biomolecules involved in different pathologies, GNP-based assays still have an advantage over many colorimetric and even fluorescence assays that use fluorophores because they readily lose their fluorescence intensity.

\section{Underexplored properties of GNPs for new enzyme assays}

Over the last few years, a great variety of morphologically different GNPs have been prepared, such as rods, urchins, prisms, wires, disks, and stars, with varying optical properties according to their size, shape, and surface chemistry [32]. In contrast to the well-studied and most frequently used spherical particles, many of these other-shaped particles have optical resonances in the near- infrared spectral range, allowing deeper penetration within tissues and an absence of photochemical damage; these features are highly advantageous for intracellular or in vivo imaging applications. Surprisingly none of the currently reported GNP-based enzyme assays takes advantage of these favorable optical characteristics. Only a few designs utilizing nanorods [33,34] or nanocages [14] have been applied to optimize the sensitivity of GNP-based assays by exploiting the tunability of surface plasmon absorption peak.

Another interesting optical feature of GNPs (especially prominent in gold nanorods and other anisotropic shapes) is their two-photon luminescence (TPL). TPL imaging, which is superior to dark-field imaging in terms of the signal-to-noise ratio, is an attractive option for intracellular imaging, in which a strong background signal often hampers the detection of low GNP concentrations. TPL imaging also allows the use of light of relatively low energy, which is less likely to cause tissue damage. TPL has mainly

Table 2. GNP-based assays for various enzymes

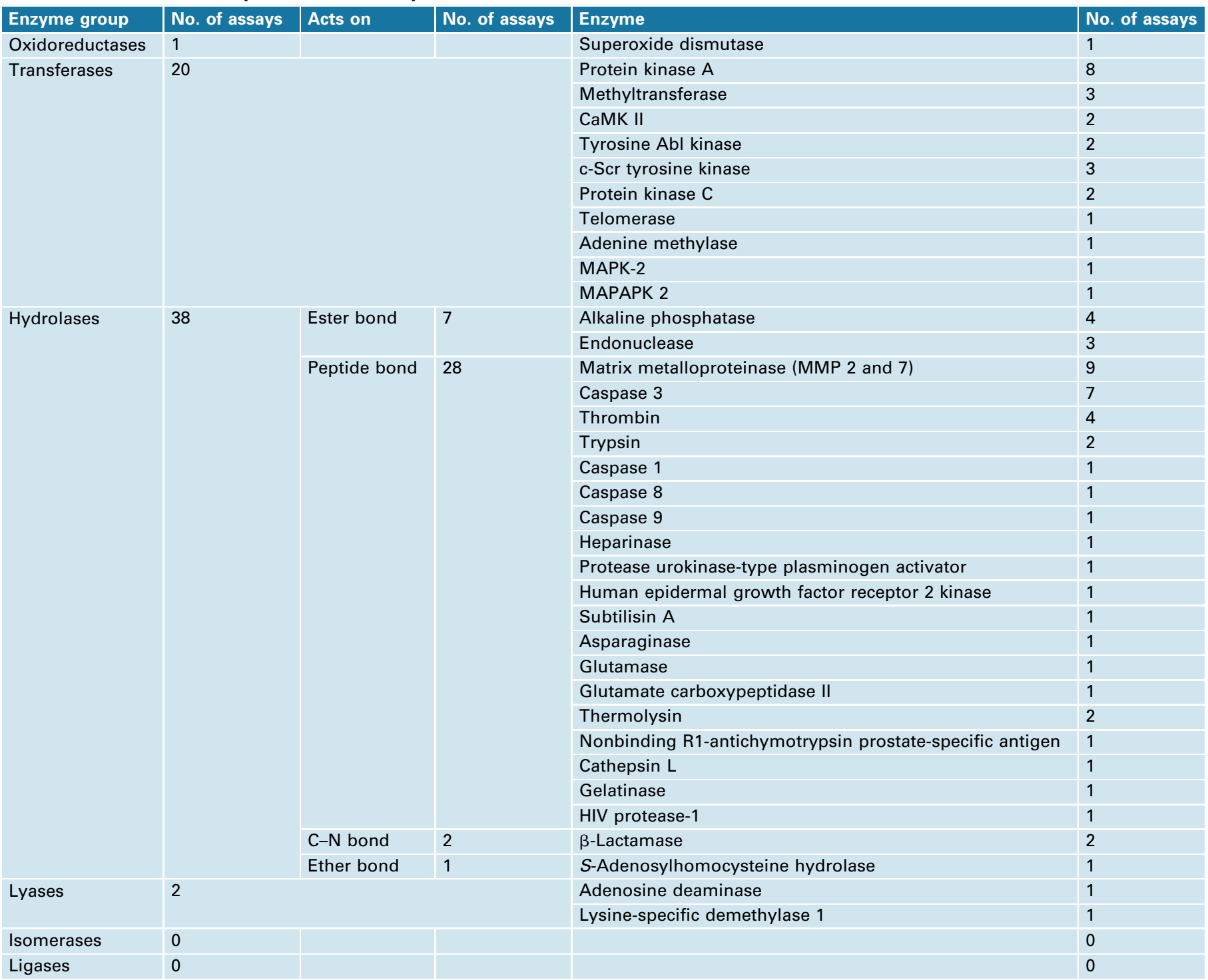


been used for bioimaging [35] and to the best of our knowledge there is no enzyme assay that uses this particular feature of GNPs.

In addition to two-photon luminescence, luminescent GNPs with high quantum yields have recently been developed [9]. The emission mechanisms have been partly elucidated and a variety of applications (chemical sensing and bioimaging) of these GNPs have been demonstrated. These particles should make very interesting candidates to replace QDs or organic fluorophores.

A fairly recently reported and underexploited GNP-based detection method is plasmonic resonance energy transfer (PRET)-based molecular imaging [36]. Choi et al. were the first to note that when the frequency of the electronic absorption band of a molecule matches the resonance frequency of the Rayleigh scattering of the plasmonic nanoparticles to which they are conjugated, energy transfer occurs between them that gives rise to distinguishable resonance-quenching dips in the Rayleigh scattering spectrum [36]. PRET-based ultrasensitive biomolecular absorption spectroscopy for single metallic nanoparticles could be used for molecular imaging such as activity measurements for a small number of functional enzymes.

Finally, there is compelling evidence showing that colloidal gold nanoparticles without any solid support exhibit intrinsic catalytic activity for some reactions like CO oxidation, aerobic oxidation of alcohols and diols, borohydride reductions and carbon-carbon cross coupling reactions [37]. Although these catalytic properties of GNPs are not yet fully understood, they could be used for the design of novel sensors.

\section{Neglected enzymes in the field of sensors}

Although there are plenty of enzymes of biological interest, only a few (kinases, MMPs, and caspases) appear frequently in the GNP-based assays reported to date (Table 2). The majority of nanosensors have been developed for proteases such as MMPs and caspases because these enzymes have been thoroughly characterized with respect to their biochemical identity, enzyme activity, and substrate specificity. In fact, the substrate preferences of proteases have been reduced to synthetic peptide sequences that are optimized for highly sensitive and specific cleavage reactions and are easy to incorporate into GNP probes [38]. There are, however, other enzymes (e.g., topoisomerases, monoacylglycerol lipase, argininosuccinate synthase) that are critical indicators of specific pathological processes, yet their activity has rarely been considered for GNP-based enzyme assays. A focus on these underinvestigated enzyme group (isomerases, lipases, and synthetases) should not only broaden the spectrum of GNP-based enzyme assays but also stimulate the development of GNP-based assays for new targets.

\section{Concluding remarks}

The number of GNP-based enzyme assays published to date is relatively low $(<100)$. One particular approach, colorimetric detection, is emerging as the leading GNPbased enzyme assay, followed closely by FRET-based assays. However, existing methods for such assays are still subject to challenges, such as the possibility of ligand exchange, nonspecific interactions with biomacromolecules, and difficulties posed by in vivo applications. The sensitivity of GNP-based assays could benefit from ratiometric measurements whenever possible. There is also plenty of room for innovation, because many of the interesting features of GNPs have not yet been fully exploited for the determination of enzyme activity, for shape-related optical differences, for luminescence and multi-photon luminescence, for plasmon resonance energy transfer, and for catalytic activities.

\section{Acknowledgment}

This work was supported by the Canadian Institutes of Health Research (CIHR-2012 MOP-119425) and the Natural Sciences and Engineering Research Council of Canada (NSERC STPGP/350345-2007). The authors thank Dr Jack Diamond for editorial suggestions.

\section{References}

1 Hayat, M.A. (ed.) (1989) Colloidal Gold, Principles, Methods, and Applications, Academic Press

2 Dykman, L. and Khlebtsov, N. (2012) Gold nanoparticles in biomedical applications: recent advances and perspectives. Chem. Soc. Rev. 41, $2256-2282$

3 Saha, K. et al. (2012) Gold nanoparticles in chemical and biological sensing. Chem. Rev. 112, 2739-2779

4 Zeng, S. et al. (2011) A review on functionalized gold nanoparticles for biosensing applications. Plasmonics 6, 491-506

5 Zhen, Z. et al. (2012) Enzymatic immuno-assembly of gold nanoparticles for visualized activity screening of histone-modifying enzymes. Anal. Chem. 84, 3614-3620

6 Laromaine, A. et al. (2007) Protease-triggered dispersion of nanoparticle assemblies. J. Am. Chem. Soc. 129, 4156-4157

7 Rosenberg, G.A. (2009) Matrix metalloproteinases and their multiple roles in neurodegenerative diseases. Lancet Neurol. 8, 205-216

8 Krizkova, S. et al. (2011) Assays for determination of matrix metalloproteinases and their activity. Trends Anal. Chem. 30, 1819-1832

9 Kim, J.H. and Chung, B.H. (2010) Proteolytic fluorescent signal amplification on gold nanoparticles for a highly sensitive and rapid protease assay. Small 6, 126-131

10 Lohse, M.J.et al. (2012) Fluorescence/bioluminescence resonance energy transfer techniques to study G-protein-coupled receptor activation and signaling. Pharmacol. Rev. 64, 299-336

11 Albizu, L. et al. (2010) Time-resolved FRET between GPCR ligands reveals oligomers in native tissues. Nat. Chem. Biol. 6, 587-594

12 Mayilo, S. et al. (2009) Long-range fluorescence quenching by gold nanoparticles in a sandwich immunoassay for cardiac troponin $\mathrm{T}$. Nano Lett. 9, 4558-4563

13 Ray, P.C. et al. (2006) Gold nanoparticle based FRET assay for the detection of DNA cleavage. J. Phys. Chem. B 110, 20745-20748

14 Xia, X. et al. (2011) An enzyme-sensitive probe for photoacoustic imaging and fluorescence detection of protease activity. Nanoscale 3, 950-953

15 Lee, S. et al. (2008) A near-infrared-fluorescence-quenched goldnanoparticle imaging probe for in vivo drug screening and protease activity determination. Angew. Chem. Int. Ed. Engl. 47, 2804-2807

16 Wang, J. et al. (2012) Visualizing human telomerase activity with primer-modified Au nanoparticles. Small 8, 259-264

17 Jun, Y-W. et al. (2009) Continuous imaging of plasmon rulers in live cells reveals early-stage caspase-3 activation at the single-molecule level. Proc. Natl. Acad. Sci. U.S.A. 106, 17735-17740

$18 \mathrm{Li}$, Y. et al. (2010) Highly sensitive electrochemical detection of human telomerase activity based on bio-barcode method. Biosens. Bioelectron. $25,2543-2547$

19 Kerman, K. and Kraatz, H-B. (2009) Electrochemical detection of protein tyrosine kinase-catalysed phosphorylation using gold nanoparticles. Biosens. Bioelectron. 24, 1484-1489

$20 \mathrm{Xu}$, S. et al. (2010) Highly sensitive electrogenerated chemiluminescence biosensor in profiling protein kinase activity and inhibition using gold nanoparticles as signal transduction probes. Anal. Chem. 82, 9566-9572

21 Forster, R.J. et al. (2009) Electrogenerated chemiluminescence. Annu. Rev. Anal. Chem. 2, 359-385

22 Asami, Y. et al. (2011) A simple set-and-mix assay for screening of protein kinase inhibitors in cell lysates. Anal. Biochem. 418, 44-49 
23 Oishi, J. et al. (2008) High-throughput colorimetric detection of tyrosine kinase inhibitors based on the aggregation of gold nanoparticles. Anal. Biochem. 373, 161-163

24 Ingram, A. et al. (2008) SERRS-based enzymatic probes for the detection of protease activity. J. Am. Chem. Soc. 130, 11846-11847

$25 \mathrm{Mu}$, C.J. et al. (2010) Self-assembled gold nanoparticle molecular probes for detecting proteolytic activity in vivo. ACS Nano 4, 1511-1520

26 Oishi, M. et al. (2009) A smart nanoprobe based on fluorescencequenching PEGylated nanogels containing gold nanoparticles for monitoring the response to cancer therapy. Adv. Funct. Mater. 19, 827-834

27 Hong, R. et al. (2006) Glutathione-mediated delivery and release using monolayer protected nanoparticle carriers. J. Am. Chem. Soc. 128, 1078-1079

28 Duchesne, L. et al. (2008) Robust ligand shells for biological applications of gold nanoparticles. Langmuir 24, 13572-13580

29 Free, P. et al. (2009) PEGylation modulates the interfacial kinetics of proteases on peptide-capped gold nanoparticles. Chem. Commun. (Camb.) 45, 5009-5011

30 Romani, B. et al. (2010) Functions of Tat: the versatile protein of human immunodeficiency virus type 1. J. Gen. Virol. 91, 1-12

31 Maysinger, D. et al. (2010) Subcellular fate of nanodelivery systems. In Organelle-Specific Pharmaceutical Nanotechnology (Weissig, V. and D’Souza, G.G., eds), pp. 93-121, John Wiley \& Sons

32 Wang, Z. et al. (2006) Kinase-catalyzed modification of gold nanoparticles: a new approach to colorimetric kinase activity screening. J. Am. Chem. Soc. 128, 2214-2215

33 Zhao, Z. et al. (2012) Highly sensitive protein kinase activity assay based on electrochemiluminescence nanoprobes. Biosens. Bioelectron. 31, 299-304

34 Kitazaki, H. et al. (2012) A colorimetric assay of protein kinase activity based on peptide-induced coagulation of gold nanorods. Colloids Surf. B: Biointerfaces 99, 7-11

35 Chen, Y. et al. (2012) Gold nanocages as contrast agents for two-photon luminescence endomicroscopy imaging. Nanomedicine 8, 1267-1270

36 Choi, Y. et al. (2009) Plasmon resonance energy transfer (PRET)based molecular imaging of cytochrome $c$ in living cells. Nano Lett. 9, $85-90$

37 Mikami, Y. et al. (2013) Catalytic activity of unsupported gold nanoparticles. Catal. Sci. Technol. 3, 58-69

38 Welser, K. et al. (2011) Protease sensing with nanoparticle based platforms. Analyst 136, 29-41

39 Liu, T. et al. (2009) Novel method to detect DNA methylation using gold nanoparticles coupled with enzyme-linkage reactions. Anal. Chem. 82, 229-233

40 Song, G. et al. (2009) A simple, universal colorimetric assay for endonuclease/methyltransferase activity and inhibition based on an enzyme-responsive nanoparticle system. ACS Nano 3, 1183-1189

41 Oishi, J. et al. (2007) Measurement of homogeneous kinase activity for cell lysates based on the aggregation of gold nanoparticles. Chembiochem 8, 875-879

42 Choi, Y. et al. (2007) Sensing phosphatase activity by using gold nanoparticles. Angew. Chem. Int. Ed. Engl. 46, 707-709

43 Serizawa, T. et al. (2010) Detection of enzyme activities based on the synthesis of gold nanoparticles in HEPES buffer. Mol. Biosyst. 6, $1565-1568$

$44 \mathrm{Xu}, \mathrm{X}$. et al. (2007) A gold-nanoparticle-based real-time colorimetric screening method for endonuclease activity and inhibition. Angew. Chem. Int. Ed. Engl. 119, 3538-3540

45 Chuang, Y-C. et al. (2012) Aqueous zymography screening of matrix metalloproteinase activity and inhibition based on colorimetric gold nanoparticles. Biosens. Bioelectron. 32, 24-31

46 Kim, G.B. et al. (2013) Colorimetric assay of matrix metalloproteinase activity based on metal-induced self-assembly of carboxy gold nanoparticles. Biosens. Bioelectron. 41, 833-839

47 Cheng, W. et al. (2011) Ultrasensitive scanometric strategy for detection of matrix metalloproteinases using a histidine tagged peptide-Au nanoparticle probe. Chem. Commun. (Camb.) 47, 2877-2879

48 Ban, Z. et al. (2008) A simple assay to probe disease-associated enzyme activity using glycosaminoglycan-assisted synthesized gold nanoparticles. Org. Biomol. Chem. 6, 4290-4292

49 Guarise, C. et al. (2006) Gold nanoparticles-based protease assay. Proc. Natl. Acad. Sci. U.S.A. 103, 3978-3982
50 Chuang, Y-C. et al. (2010) An optical biosensing platform for proteinase activity using gold nanoparticles. Biomaterials 31, 6087-6095

51 Pan, Y. et al. (2012) Colorimetric detection of apoptosis based on caspase-3 activity assay using unmodified gold nanoparticles. Chem. Commun. (Camb.) 48, 997-999

52 Jiang, T. et al. (2009) Colorimetric screening of bacterial enzyme activity and inhibition based on the aggregation of gold nanoparticles. Chem. Commun. (Camb.) 45, 1972-1974

53 Lin, J-H. et al. (2010) Colorimetric assay for $S$-adenosylhomocysteine hydrolase activity and inhibition using fluorosurfactant-capped gold nanoparticles. Anal. Chem. 82, 8775-8779

54 Zhang, L. et al. (2012) Enzyme-regulated unmodified gold nanoparticle aggregation: a label free colorimetric assay for rapid and sensitive detection of adenosine deaminase activity and inhibition. Chem. Commun. (Camb.) 48, 10996-10998

55 Piao, J.Y. and Chung, D.S. (2012) Novel colorimetric assay of LSD1 activity using gold nanoparticles. Analyst 137, 2669-2673

56 Jang, B. et al. (2011) Gold nanorod-photosensitizer complex for nearinfrared fluorescence imaging and photodynamic/photothermal therapy in vivo. ACS Nano 5, 1086-1094

$57 \mathrm{Kim}, \mathrm{Y}-\mathrm{P}$. et al. (2010) Bioluminescent nanosensors for protease detection based upon gold nanoparticle-luciferase conjugates. Chem. Commun. (Camb.) 46, 76-78

58 Park, S. et al. (2012) Gold nanoparticle-based fluorescence quenching via metal coordination for assaying protease activity. Gold Bull. 45 , 213-219

59 Lowe, S.B. et al. (2011) Multiplex sensing of protease and kinase enzyme activity via orthogonal coupling of quantum dot-peptide conjugates. ACS Nano 6, 851-857

60 Zhen, S.J. et al. (2008) Tryptophan-contained peptide-functional nanomaterials as general spectrofluorometric reagents for enzyme. Talanta 76, 230-232

61 Park, K. et al. (2012) Cascade imaging of proteolytic pathways in cancer cells using fluorescent protein-conjugated gold nanoquenchers. Chem. Commun. (Camb.) 48, 10547-10549

62 Sun, I-C. et al. (2010) Caspase sensitive gold nanoparticle for apoptosis imaging in live cells. Bioconjug. Chem. 21, 1939-1942

$63 \mathrm{Kim}$, Y-P. et al. (2008) Energy transfer-based multiplexed assay of proteases by using gold nanoparticle and quantum dot conjugates on a surface. Anal. Chem. 80, 4634-4641

64 Koteshwara Reddy, K. and Vengatajalabathy Gobi, K. (2012) Activated direct electron transfer of nanoAu bioconjugates of cytochrome $\mathrm{c}$ for electrocatalytic detection of trace levels of superoxide dismutase enzyme. Electrochim. Acta 78, 109-114

$65 \mathrm{He}$, X. et al. (2011) A sensitive signal-on electrochemical assay for MTase activity using AuNPs amplification. Biosens. Bioelectron. 28, 298-303

$66 \mathrm{Xu}$, X. et al. (2009) A DNA-based electrochemical strategy for label-free monitoring the activity and inhibition of protein kinase. Chem. Commun. (Camb.) 45, 6946-6948

67 Wang, Z. et al. (2005) Microarray-based detection of protein binding and functionality by gold nanoparticle probes. Anal. Chem. 77, 5770-5774

68 Kim, Y-P. et al. (2007) Protein kinase assay on peptide-conjugated gold nanoparticles by using secondary-ion mass spectrometric imaging. Angew. Chem. Int. Ed. Engl. 46, 6816-6819

69 Kim, Y-P. et al. (2008) Protein kinase assay on peptide-conjugated gold nanoparticles. Biosens. Bioelectron. 23, 980-986

70 Wang, G. et al. (2013) Detection of T4 polynucleotide kinase activity with immobilization of $\mathrm{TiO}_{2}$ nanotubes and amplification of $\mathrm{Au}$ nanoparticles. Biosens. Bioelectron. 43, 125-130

71 Ruan, C. et al. (2006) Detection of alkaline phosphatase using surfaceenhanced Raman spectroscopy. Anal. Chem. 78, 3379-3384

72 Jiang, Z. et al. (2012) A sensitive enzyme-catalytic nanogold-resonance scattering spectral assay for alkaline phosphate. Bioprocess Biosyst. Eng. 35, 781-787

73 Bonomi, R. et al. (2011) Detection of enzyme activity through catalytic signal amplification with functionalized gold nanoparticles. Angew. Chem. Int. Ed. Engl. 50, 2307-2312

74 Mahmoud, K.A. and Luong, J.H.T. (2008) Impedance method for detecting HIV-1 protease and screening for its inhibitors using ferrocene-peptide conjugate/Au nanoparticle/single-walled carbon nanotube modified electrode. Anal. Chem. 80, 7056-7062

75 Cao, Y. et al. (2013) A simple and general approach to assay protease activity with electrochemical technique. Biosens. Bioelectron. 45, 1-5 